\title{
Synthesis and Pharmacological Screening of Some Benzoxazole Derivatives as Anti-inflammatory Agents
}

\author{
Mohammed Rageeb Mohammed Usman ${ }^{*}$ Rana Sohil D ${ }^{1}$, Md. Abullais Md. \\ Usman $^{1}$,Shaikh TanverY ${ }^{2}$,Sayad Imran wahab ${ }^{3}$ \\ ${ }^{*}$ Assistant Professor, Department of Pharmacognosy, Smt. S. S. Patil College of Pharmacy, Chopda, Maharashtra, India \\ ${ }^{2}$ Assistant Professor, Department of Pharmaceutics, Smt. S. S. Patil College of Pharmacy, Chopda, Maharashtra, India \\ ${ }^{3}$ Lecturer, Department of Pharmaceutical Chemistry, Y. B. Chavan College of Pharmacy, Aurangabad, Maharashtra, India
}

\begin{abstract}
A series of some new 5-substituted benzoxazoles were synthesized and were characterized by different methods like IR, ${ }^{1}$ HNMR and MASS spectra. After conformation of structure assigned, these compounds were screened for its antiinflammatory activity.
\end{abstract}

Keywords: Benzoxazole, Anti-inflammatory, Pharmacological, Derivatives, Phytochemical.

\section{INTRODUCTION}

Inflammation evidence of many diseases is major concern for physicians throughout the word. The single most important event in this process is accumulation of large number of phagocytic cells of the site of the inflammation. Tissue injury caused by introduction of a foreign antigen, trauma, or local exposure to certain chemicals triggers complex processes of inflammation. This may consists of a fluid stasis as well as the accumulation of several cellular and no cellular elements of the immune response [1-6].

In most of these cases, it has been proved that the 5-substituted benzoxazole [7], substituted sulfonyl derivatives [8] and carbohydrazides [9], have promising anti-inflammatory activity. Also benzoxazole at its $5^{\text {th }}$ position [10], is more prone for its lipophilic action and therefore we go the substitution at $5^{\text {th }}$ position of benzoxazole. Hence, it was planned to synthesize the $N^{\top}$ [substituted sulfonyl]-1,3-benzoxazole-5-carbohydrazide to get good antiinflammatory activity.

In the present investigation, series of $N$ [substituted sulfonyl]-1,3-benzoxazole-5-carbohydrazide, were synthesized using appropriate synthetic route (Scheme: I page no.3) and were screened for its antiinflammatory activity (VIa-VIh).

4-Hydroxy-3-nitro-benzoic acid methyl ester (II) was synthesized in an excellent yield by electrophilic substitution, nitration on p-hydroxy methyl benzoate (I) by concentrated nitric acid and concentrated sulfuric acid. Compound (II) on reduction with the help of reducing agent like sodium dithionate [11], with alcohol afforded 3-amino-4-hydroxy-benzoic acid methyl ester (III). Reaction of compound (III) with two appropriate aliphatic acids such as, formic acid and acetic acid gives corresponding 2-subtituted benzoxazole-5-carboxylic acid methyl esters (IVa and IVb). The reaction of compounds (IV) with hydrazine hydrate in ethanol on refluxing gives the corresponding 2-substituted benzoxazole-5-carboxylic acid hydrazides $(\mathrm{Va}$ and $\mathrm{Vb}$ ) is the nucleophilic substitution type reaction. On further reaction of compounds (V) with the different nucleophilic substitution of substituted sulfonyl chloride derivatives afforded the corresponding eight $N$ [substituted sulfonyl]-1,3-benzoxazole-5-carbohydrazide (VIa-VIh).

The purity and homogeneity of compounds synthesized were determined by their sharp melting points, TLC, IR spectra.

Preliminary pharmacological screening was performed, which includes approximate toxicity testing $\left(\mathrm{LD}_{50}\right)$ [13] and antiinflammatory activity [14]. The $\mathrm{LD}_{50}$ of the test compounds performed on the rats as per the OECD 423 guidelines for selection of dose.<smiles>[R5]S(=O)(=O)NNC(=O)c1ccc2oc(Br)nc2c1</smiles>

Figure 1: $N$ [substituted sulfonyl]-1,3-benzoxazole-5-carbohydrazide (VI) 
IOSR Journal of Pharmacy

Vol. 2, Issue 3, May-June, 2012, pp.374-379

Table 1: Different substituted compounds

\begin{tabular}{lll}
\hline Compound & $\mathbf{R 1}$ & $\mathbf{R 2}$ \\
\hline VIa & $\mathrm{H}$ & 4-methyl phenyl \\
VIb & $\mathrm{CH}_{3}$ & 4-methyl phenyl \\
VIc & $\mathrm{H}$ & 4-aceta amido phenyl \\
VId & $\mathrm{CH}_{3}$ & 4-aceta amido phenyl \\
VIe & $\mathrm{H}$ & 4-chloro phenyl \\
VIf & $\mathrm{CH}_{3}$ & 4-chloro phenyl \\
VIg & $\mathrm{H}$ & Benzene \\
VIh & $\mathrm{CH}_{3}$ & Benzene \\
\hline
\end{tabular}

Scheme I:<smiles>CC(=O)c1ccc(O)cc1</smiles>

( I )<smiles>COC(=O)c1ccc(O)c([N+](=O)[O-])c1</smiles>

( II )
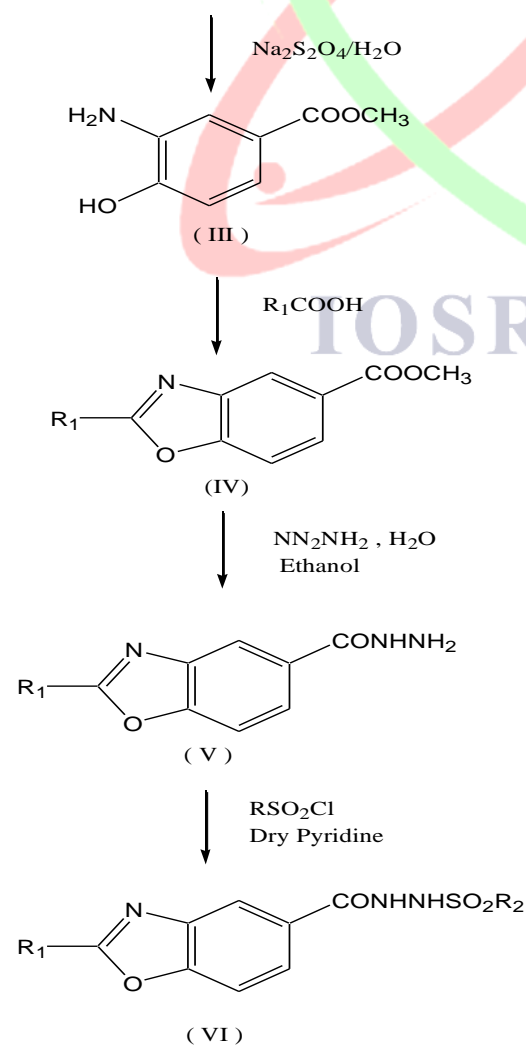

\section{MATERIAL AND METHODS}


All chemicals were used as purchased pure from Hi-Media, E-Merck. p-hydroxy methyl benzoate (I) was used as starting material undergoes electrophilic substitution reaction, nitration by using concentrated nitric acid and concentrated sulphuric acid gives 4-Hydroxy-3-nitro-benzoic acid methyl ester (II) this reaction is carried out at $0-10^{\circ} \mathrm{C}$ and recrystallised by methanol. Compound (II) undergoes reduction by using sodium dithionate as reducing agent in mixture with methanol gives good yield of 3-amino-4-hydroxy-benzoic acid methyl ester (III). This was recrystallised by using methanol. On further reaction of compound (III) with aliphatic acid like formic and acetic acid gives corresponding compounds 2-subtituted benzoxazole-5-carboxylic acid methyl ester (IVa-IVb). Both these products were recrystallised from alcohol. Compounds $(\mathrm{IVa}-\mathrm{IVb})$ on reaction with hydrazine hydrate and mixture with ethanol gives corresponding 2-substituted benzoxazole-5carboxylic acid hydrazides $(\mathrm{Va}-\mathrm{Vb})$ also both these products was recrystallised from alcohol. And finally compounds (Va$\mathrm{Vb}$ ) on further reaction with substituted sulfonyl chlorides by using pyridine as catalyst which traps $\mathrm{HCl}$ gas in compounds gives corresponding $N$ [substituted sulfonyl]-1,3-benzoxazole-5-carbohydrazide (VIa-VIh) compounds. Finally these eight compounds were recrystallised by ethanol give pure compounds.

The melting points of the compounds were determined in open capillary method which was uncorrected.

Porous silica gel plates activated at $110^{\circ} \mathrm{C}$ for $30 \mathrm{~min}$. were used for thin layer chromatography (TLC) and were developed with iodine vapours. IR spectra of compounds were recorded using $\mathrm{KBr}$ pellets on FTIR. ${ }^{1} \mathrm{H}-\mathrm{NMR}$ spectra (solvents) were recorded on Varian EM 390 spectra (chemicals shift in $\delta \mathrm{ppm}$ ). Mass Spectra of the synthesized compounds were recorded on (FAB-MS) [11-12].

\section{RESULT AND DISCUSSION}<smiles>[R]S(=O)(=O)NNC(=O)c1ccc2oc(Br)nc2c1</smiles>

Figure 2: $N$ [substituted sulfonyl]-1,3-benzoxazole-5-carbohydrazide (VI)

Table 2: The results studies are summarized as

\begin{tabular}{llllll} 
Compound No. & R & Molecular Formula & $\begin{array}{l}\text { Mp }\left({ }^{0} \mathbf{C}\right) \\
\text { uncorrected }\end{array}$ & Yield (\%) & $\mathbf{R}_{\mathbf{f}}$ Value \\
\hline VIa & Tosyl & $\mathrm{C}_{15} \mathrm{H}_{13} \mathrm{~N}_{3} \mathrm{O}_{4} \mathrm{~S}$ & $72-74$ & $38 \%$ & 0.63 \\
VIb & Tosyl & $\mathrm{C}_{16} \mathrm{H}_{15} \mathrm{~N}_{3} \mathrm{O}_{4} \mathrm{~S}$ & $78-80$ & $57 \%$ & 0.60 \\
VIc & p-aceta amido & $\mathrm{C}_{16} \mathrm{H}_{14} \mathrm{~N}_{4} \mathrm{O}_{5} \mathrm{~S}$ & $92-94$ & $72 \%$ & 0.57 \\
VId & p-aceta amido & $\mathrm{C}_{17} \mathrm{H}_{16} \mathrm{~N}_{4} \mathrm{O}_{5} \mathrm{~S}$ & $88-90$ & $40 \%$ & 0.54 \\
VIe & p-chloro & $\mathrm{C}_{14} \mathrm{H}_{10} \mathrm{ClN}_{3} \mathrm{O}_{4} \mathrm{~S}$ & $110-112$ & $75 \%$ & 0.53 \\
VIf & p-chloro & $\mathrm{C}_{15} \mathrm{H}_{12} \mathrm{ClN}_{3} \mathrm{O}_{4} \mathrm{~S}$ & $102-104$ & $72 \%$ & 0.64 \\
VIg & Benzene & $\mathrm{C}_{14} \mathrm{H}_{11} \mathrm{~N}_{3} \mathrm{O}_{4} \mathrm{~S}$ & $69-70$ & $30 \%$ & 0.62 \\
VIh & Benzene & $\mathrm{C}_{15} \mathrm{H}_{13} \mathrm{~N}_{3} \mathrm{O}_{4} \mathrm{~S}$ & $66-68$ & $40 \%$ & 0.72 \\
\hline
\end{tabular}

Elemental analysis of the given compounds is summarized below. 
IOSR Journal of Pharmacy

Vol. 2, Issue 3, May-June, 2012, pp.374-379

All products were isolated and characterized by spectrometric methods $\left({ }^{1} \mathrm{H}\right.$ NMR, IR and mass spectra). A detailed IR, NMR and mass analysis is explained below:

Compound VIa $\left(\mathrm{C}_{15} \mathrm{H}_{13} \mathrm{~N}_{3} \mathrm{O}_{4} \mathrm{~S}\right)$

IR (KBr) cm cm $^{-1} 3390$ (-NH-str.), 1315 and 1398 (-S-O str.), 1730 (-CO-str.), 1625 (C-N str.), 3085 (Ar-H str.), 835 (C=C bending), $1165 \mathrm{~cm}^{-1}$ (ether group in ring);

${ }^{1}$ H NMR: $\left(\mathrm{CDCl}_{3}\right) \delta 7.34-7.95(\mathrm{~m}, \mathrm{Ar}-\mathrm{H}, 4 \mathrm{H} \times 2), \delta 8.0(\mathrm{~s}, \mathrm{NH}, 2 \mathrm{H}), \delta 2.35\left(\mathrm{~d}, \mathrm{CH}_{3}, 3 \mathrm{H}\right)$.

FAB-MS: $(\mathrm{m} / \mathrm{z}, 100 \%): 331\left(\left[\mathrm{M}^{+}\right],(100 \%)\right.$

Elemental analysis (\%): Calculated: C: $52.9, \mathrm{H}: 4.04, \mathrm{~N}: 13.08$,

Found: C: 52.5, H: 4.04, N: 13.12 .

Compound VIb $\left(\mathrm{C}_{16} \mathrm{H}_{15} \mathrm{~N}_{\mathbf{3}} \mathbf{O}_{4} \mathrm{~S}\right)$

IR (KBr) cm ${ }^{-1}: 3319$ (-NH- str.), 1315 (-S-O str.), 1730 (-CO- str.), 1625 (C=N str.), 3010 (Ar-H str.), 2230 (C-C str.)

${ }^{1}$ H NMR: $\left(\mathrm{CDCl}_{3}\right) \delta 7.34-7.95(\mathrm{~m}, \mathrm{Ar}-\mathrm{H}, 4 \mathrm{H}+3 \mathrm{H}), \delta 8.0(\mathrm{~s}, \mathrm{NH}, 2 \mathrm{H}), \delta 2.35\left(\mathrm{~d}, \mathrm{CH}_{3}, 3 \mathrm{H} \times 2\right)$.

FAB-MS: $(\mathrm{m} / \mathrm{z}, 100 \%): 345\left(\left[\mathrm{M}^{+}\right],(100 \%)\right.$

Elemental analysis (\%): Calculated: C: $55.6, \mathrm{H}: 4.34, \mathrm{~N}: 12.07$,

Found: C: $55.5, \mathrm{H}: 4.38, \mathrm{~N}: 12.10$.

Compound VIc $\left(\mathrm{C}_{16} \mathrm{H}_{14} \mathrm{~N}_{4} \mathrm{O}_{5} \mathrm{~S}\right)$

IR (KBr) cm ${ }^{-1}$ : 3327 (-NH- str.), 1352 (-S-O str.), 1730 (-CO- str.), 1116 and1172 cm ${ }^{-1}$ (CONH str.), $3010 \mathrm{~cm}^{-1}$ (Ar-H str.).

${ }^{1}$ H NMR: $\left(\mathrm{CDCl}_{3}\right) \delta 7.44-7.95(\mathrm{~m}, \mathrm{Ar}-\mathrm{H}, 4 \mathrm{H} \times 2), \delta 8.0(\mathrm{~s}, \mathrm{NH}, 3 \mathrm{H}), \delta 2.02\left(\mathrm{~d}, \mathrm{CH}_{3}, 3 \mathrm{H}\right)$.

FAB-MS: $(\mathrm{m} / \mathrm{z}, 100 \%): 374\left(\left[\mathrm{M}^{+}\right],(100 \%)\right.$

Elemental analysis (\%): Calculated: C: $53.63, \mathrm{H}: 3.91, \mathrm{~N}: 15.64$,

Compound VId $\left(\mathrm{C}_{17} \mathrm{H}_{16} \mathrm{~N}_{4} \mathrm{O}_{5} \mathrm{~S}\right)$

Found: C: $53.65, \mathrm{H}: 3.87, \mathrm{~N}: 15.76$.

IR (KBr) cm ${ }^{-1}$ : 3324 (-NH- str.), 1322 (-S-O str.), 1730 (-CO- str.), 1629 (C=N str.), 3110 (Ar-H str.), 3180 (CONH str.).

${ }^{1}$ H NMR: $\left(\mathrm{CDCl}_{3}\right) \delta 7.44-7.95(\mathrm{~m}, \mathrm{Ar}-\mathrm{H}, 4 \mathrm{H}+3 \mathrm{H}), \delta 8.0(\mathrm{~s}, \mathrm{NH}, 3 \mathrm{H}), \delta 2.02$ and $2.35\left(\mathrm{~d}, \mathrm{CH}_{3}, 3 \mathrm{H} \times 2\right)$.

FAB-MS: $(\mathrm{m} / \mathrm{z}, 100 \%): 388\left(\left[\mathrm{M}^{+}\right],(100 \%)\right.$

Elemental analysis (\%): Calculated: C: $52.57, \mathrm{H}: 4.12, \mathrm{~N}: 14.43$,

Compound VIe $\left(\mathrm{C}_{14} \mathrm{H}_{10} \mathrm{ClN}_{3} \mathrm{O}_{4} \mathrm{~S}\right)$

Found: C: $52.560, \mathrm{H}: 4.16, \mathrm{~N}: 14.47$.

IR (KBr) cm ${ }^{-1}$ : 3216 (-NH- str.), 1339 (-S-O str.), 3090 (Ar-H str.), 772 (C-Cl str.)

${ }^{1}$ H NMR: $\left(\mathrm{CDCl}_{3}\right) \delta 7.44-7.95(\mathrm{~m}, \mathrm{Ar}-\mathrm{H}, 4 \mathrm{H} \times 2), \delta 8.0(\mathrm{~s}, \mathrm{NH}, 2 \mathrm{H})$.

FAB-MS: $(\mathrm{m} / \mathrm{z}, 100 \%): 351.50\left(\left[\mathrm{M}^{+}\right], 100 \%\right)$

Elemental analysis (\%): Calculated: C: 45.94, H: 2.94, N: 12.37 ,

Found: C: 45.97, H: 2.98, N: 12.40 .

Compound VIf $\left(\mathrm{C}_{15} \mathrm{H}_{12} \mathrm{ClN}_{3} \mathrm{O}_{4} \mathrm{~S}\right)$

IR (KBr) cm ${ }^{-1}$ : 3204 (-NH- str.), 1354 and 1329 (-S-O str.), 1730 (-CO- str.), 3180 (CONH str.), 3097 (Ar-H str.), 767 (C-Cl str.),

${ }^{1}$ H NMR: $\left(\mathrm{CDCl}_{3}\right) \delta 7.44-7.95(\mathrm{~m}, \mathrm{Ar}-\mathrm{H}, 4 \mathrm{H}+3 \mathrm{H}), \delta 8.0(\mathrm{~s}, \mathrm{NH}, 2 \mathrm{H}), \delta 2.35\left(\mathrm{~d}, \mathrm{CH}_{3}, 3 \mathrm{H}\right)$

FAB-MS: $(\mathrm{m} / \mathrm{z}, 100 \%): 317.50\left(\left[\mathrm{M}^{+}\right], 100 \%\right)$

Elemental analysis (\%): Calculated: C: $47.81, \mathrm{H}: 3.73, \mathrm{~N}: 11.81$,

Found: C: 47.78, H: 3.75, N: 11.84 .

Compound VIg $\left(\mathrm{C}_{14} \mathrm{H}_{11} \mathrm{~N}_{3} \mathrm{O}_{4} \mathrm{~S}\right)$

IR (KBr) cm cm $^{-1} 3350$ (-NH- str.), 1329 (-S-O str.), $1730{ }^{1}$ (-CO- str.), 3174 (CONH str.), 3097 (Ar-H str.), 674 (C-C bending)

${ }^{1}$ H NMR: $\left(\mathrm{CDCl}_{3}\right) \delta 7.03-7.95(\mathrm{~m}, \mathrm{Ar}-\mathrm{H}, 5 \mathrm{H}+4 \mathrm{H}), \delta 8.0(\mathrm{~s}, \mathrm{NH}, 2 \mathrm{H})$.

FAB-MS: $(\mathrm{m} / \mathrm{z}, 100 \%): 317\left(\left[\mathrm{M}^{+}\right], 100 \%\right)$

Elemental analysis (\%): Calculated: C: $51.31, \mathrm{H}: 3.28, \mathrm{~N}: 13.81$,

Found: C: 51.33, H: 3.32, N: 13.85 .

Compound VIh $\left(\mathrm{C}_{15} \mathrm{H}_{13} \mathrm{~N}_{3} \mathrm{O}_{4} \mathrm{~S}\right)$

IR (KBr) cm ${ }^{-1}$ : 3204 (-NH- str.), 1346 (-S-O str.), 1730 (-CO- str.), 1625 (C=N str.), 3085 (Ar-H str.), 2990 (C-C str.).

${ }^{1}$ H NMR: $\left(\mathrm{CDCl}_{3}\right) \delta 7.03-7.95(\mathrm{~m}, \mathrm{Ar}-\mathrm{H}, 4 \mathrm{H} \times 2), \delta 8.0(\mathrm{~s}, \mathrm{NH}, 2 \mathrm{H}), \delta 2.35\left(\mathrm{~d}, \mathrm{CH}_{3}, 3 \mathrm{H}\right)$.

FAB-MS: $(\mathrm{m} / \mathrm{z}, 100 \%): 331\left(\left[\mathrm{M}^{+}\right], 100 \%\right)$

Elemental analysis (\%): Calculated: C: 53.31, H: 3.75, N: 13.12,

Found: C: 53.34, H: 3.77, N: 13.16. 
IOSR Journal of Pharmacy

Vol. 2, Issue 3, May-June, 2012, pp.374-379

\section{Pharmacological Screening}

\section{LD50}

$\mathrm{LD}_{50}$ of test compounds was performed in National Toxicological Center, Pune and determined on mice as per the OECD Guidelines $423.2000 \mathrm{mg} / \mathrm{kg}$ dose was considered as $\mathrm{LD}_{50} .1 / 10^{\text {th }}$ of the $\mathrm{LD}_{50}$ was considered as an effective dose i.e.200 $\mathrm{mg} / \mathrm{kg}$.

\section{Anti-Inflammatory Activity}

The anti-inflammatory activities of these compounds were done by using carrageenan induced rat paw edema method described by Winter et al (1962) [10].

Table 4: Anti-inflammatory activity of Some Benzoxazole Derivatives

\begin{tabular}{|c|c|c|c|c|}
\hline \multirow[t]{2}{*}{ Group } & \multirow[t]{2}{*}{ Test Material (dose) } & \multicolumn{3}{|c|}{ Mean increase in paw volume and \% inhibition } \\
\hline & & $1 \mathrm{hr}$. & $2 \mathrm{hr}$. & $3 \mathrm{hr}$. \\
\hline 1. & Control & $1.29 \pm 0.152$ & $1.73 \pm 0.200$ & $1.90 \pm 0.116$ \\
\hline 2. & $\begin{array}{l}\text { Standard(Ibuprofen } \\
50 \mathrm{mg} / \mathrm{kg} \text { ) }\end{array}$ & $\begin{array}{l}0.95 \pm 0.158 \\
(26.35 \%)\end{array}$ & $\begin{array}{l}1.09 \pm 0.178 \\
(36.99 \%)\end{array}$ & $\begin{array}{l}1.03 \pm 0.163 \\
(45.78 \%)\end{array}$ \\
\hline 3. & $\begin{array}{l}\text { VIa } \\
200 \mathrm{mg} / \mathrm{kg}\end{array}$ & $\begin{array}{l}1.06 \pm 0.11 \\
(17.82 \%)\end{array}$ & $\begin{array}{l}1.38 \pm 0.019 \\
(20.23 \%)\end{array}$ & $\begin{array}{l}1.66 \pm 0.168 \\
(18.63 \%)\end{array}$ \\
\hline 4. & $\begin{array}{l}\text { VIb } \\
200 \mathrm{mg} / \mathrm{kg}\end{array}$ & $\begin{array}{l}1.13 \pm 0.212 \\
(12.40 \%)\end{array}$ & $\begin{array}{l}1.38 \pm 0.200 \\
(20.23 \%)\end{array}$ & $\begin{array}{l}1.51 \pm 0.292 \\
(20.52 \%)\end{array}$ \\
\hline 5. & $\begin{array}{l}\text { VIc } \\
200 \mathrm{mg} / \mathrm{kg}\end{array}$ & $\begin{array}{l}1.19 \pm 0.364 \\
(7.75 \%)\end{array}$ & $\begin{array}{l}1.24+0.167 \\
(14.45 \%)\end{array}$ & $\begin{array}{l}1.50 \pm 0.342 \\
(21.05 \%)\end{array}$ \\
\hline 6. & $\begin{array}{l}\text { VId } \\
200 \mathrm{mg} / \mathrm{kg}\end{array}$ & $\begin{array}{l}1.22+0.0740 \\
(5.42 \%)\end{array}$ & $\begin{array}{l}1.58 \pm 0.081 \\
(12.45 \%)\end{array}$ & $\begin{array}{l}1.68 \pm 0.223 \\
(11.57 \%)\end{array}$ \\
\hline 7. & $\begin{array}{l}\text { VIe } \\
200 \mathrm{mg} / \mathrm{kg}\end{array}$ & $\begin{array}{l}1.28 \pm 0.98 \\
(0.77 \%)\end{array}$ & $\begin{array}{l}1.48 \pm 0.305 \\
(14.45 \%)\end{array}$ & $\begin{array}{l}1.50 \pm 0.340 \\
(21.05 \%)\end{array}$ \\
\hline 8. & $\begin{array}{l}\text { VIf } \\
200 \mathrm{mg} / \mathrm{kg}\end{array}$ & $\begin{array}{l}1.09 \pm 0.0659 \\
(15.50 \%)\end{array}$ & $\begin{array}{l}1.35 \pm 0.100 \\
(21.96 \%)\end{array}$ & $\begin{array}{l}1.22 \pm 0.189 \\
(30.78 \%)\end{array}$ \\
\hline 9. & $\begin{array}{l}\mathrm{VIg} \\
200 \mathrm{mg} / \mathrm{kg}\end{array}$ & $\begin{array}{l}1.47 \pm 0.285 \\
(21.63 \%)\end{array}$ & $\begin{array}{l}0.57 \pm 0.158 \\
(26.35 \%)\end{array}$ & $\begin{array}{l}1.07 \pm 0.328 \\
(40.05 \%)\end{array}$ \\
\hline 10. & $\begin{array}{l}\text { VIh } \\
200 \mathrm{mg} / \mathrm{kg}\end{array}$ & $\begin{array}{l}1.10 \pm 0.0815 \\
(14.72 \%)\end{array}$ & $\begin{array}{l}1.22 \pm 0.169 \\
(35.78 \%)\end{array}$ & $\begin{array}{l}1.00 \pm 0.145 \\
(42.19 \%)\end{array}$ \\
\hline
\end{tabular}




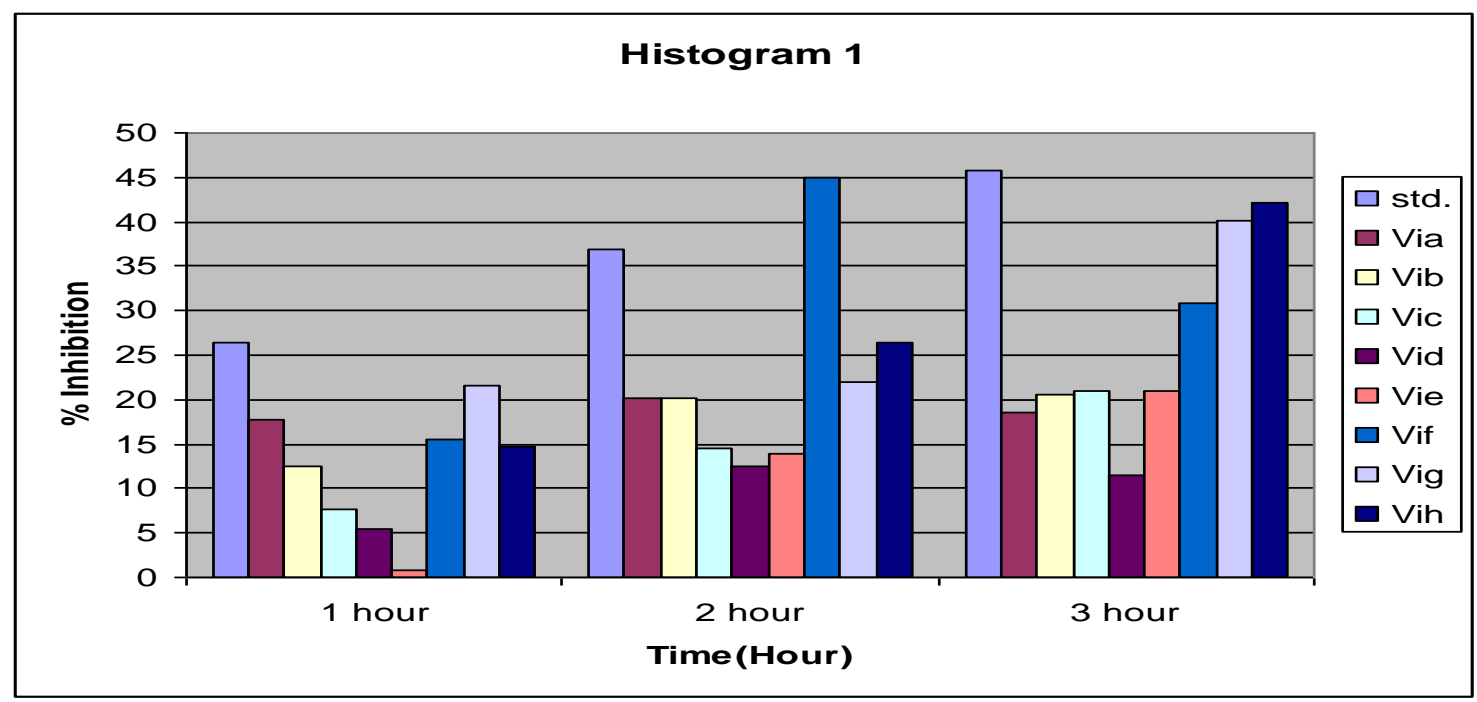

Figure 3: Anti-inflammatory activity of Some Benzoxazole Derivatives

\section{CONCLUSION}

5-substituted benzoxazole has proved to be a promising moiety for antiinflammatory activity. The test compounds (VIa-VIh) showed significant antiinflammatory activity compared with the standard drug Ibuprofen. Among these compounds VId and VIe possesses good and compound VIb, VIc and VIf possess moderate antiinflammatory activity. All the significant compounds also possess antiinflammatory activity with reduced toxicity.

The compounds can be further exploited for testing other pharmacological activities. QSAR parameters can be added in the present study shall help to as certain proposed and observed activity.

\section{REFERENCES}

1. M. J. Brown, and Bennet DN, Clinical pharmacology, 2003, 276-280.

2. Goodman, and G. J. Hardman, Pharmacological Basis of Therapeutics 2001. 687.

3. J. G. Lombardino, Non steroidal anti-inflammatory Drugs, John Wiley and Sons. Inc. (Ltd), 781, 1985, 116128.

4. H. Mohan, Text book of pathology, 4, New Delhi, 2000, 132.

5. C. A. Gyton, and J. E. Hall, Textbook of Medical Physiology, 9, 1996, 439.

6. M. Koksal, N. Ghokhan, and E. Kupeli, Arch. Pharm (Weinheim) 3, 2005, 117-25.

7. A. Mohd, S. Kumar, Indian Drugs, 42(2), 2005, 73-77.

8. krapcho John, United States Patent, 4156079, 1979.

9. Y. Sato, M. Yamada, and K. Kobayashi, Chem. Pharm. Bull, 46(3), 1998, 445-451.

10. M. Peter, and Pojer, Aust. J. Chem., 32, 1979, 201-4.

11. B. Gopalkrishna, N. Raghunandan, and V, Rao, Indian drugs, 42, 2005, 369-374.

12. B. Furniss, Vogel's text book of Practical Organic Chemistry, 5, 2004, 1071.

13. Guidelines Document: Testing and Assessment no. AOT 425, 2001.

14. C. A. Winter, E. Risely, and E. Nuss, Proc. Soc. Exp. Bio \& Med., 111, 1962, 544. 